(c) American Dairy Science Association, 2003.

\title{
Application of Fluorescence Spectroscopy and Chemometrics in the Evaluation of Processed Cheese During Storage
}

\author{
J. Christensen*, V. T. Povlsen*, and J. Sørensen† \\ *Food Technology, Department of Dairy and Food Science, \\ The Royal Veterinary and Agricultural University, \\ Rolighedsvej 30, DK-1958 Frederiksberg C, Denmark \\ †Arla Foods Innovation, Søderupvej 26, DK-6920 Videbæk, Denmark
}

during storage. Common stress factors in the distribution retails and production are light exposure and varying temperature, which can result in reduced shelf life partly due to increased formation of free radicals. Therefore, processed cheese samples stored under different light and heat conditions are investigated in the present study.

Many methods have been developed to shed light on the degree of oxidation of dairy products, a process that consists of several stages. The early stage of lipid oxidation can form hydroperoxides, which normally are measured by HPLC or by evaluation of the peroxide value (Emmons et al., 1986). Secondary oxidation products can be analyzed by static or dynamic headspace GCMS (Sunesen et al., 2002) or methods using thiobarbituric acid (Kristensen et al., 2001). Methods based on electron spin resonance spectrometry were recently suggested for monitoring the formation of radicals during the oxidation of processed cheese (Kristensen and Skibsted, 1999). All these methods for evaluation of the oxidative levels of dairy products have in common, that they are destructive and time consuming. In this study, the potential of front face fluorescence, measured directly on the cheese surface were investigated, as an alternative, fast and nondestructive method. Theoretically the potential of fluorescence seems sound, since the cheese product contains well known fluorescent compounds in form of aromatic amino acids, vitamin A and riboflavin (Duggan et al., 1957), which all have been reported to be affected during structural changes in cheese (Dufour et al., 2001) or during light and heat exposure (Kristensen et al., 2001; Whited et al., 2002; Wold et al., 2002).

Fluorescence spectroscopy is a sensitive, rapid and noninvasive analytical technique that can provide information on the presence of fluorescent molecules and their environment in all sorts of biological samples. The development and improvement of chemometric methods (Bro, 1996; Bro, 1997; Andersson and Bro, 2000) combined with the technical and optical development of spectrofluorometers have in recent years increased the possibilities for the use of fluorescence spectroscopy.

Received September 2, 2002

Accepted October 25, 2003.

Corresponding author: Jakob Christensen; e-mail: jach@kvl.dk. 
Thus, online monitoring sensors that enable measurements of complete excitation emission spectra (fluorescence landscapes) are now commercially available.

In the last years, a few studies have focused on the potential of using front face fluorescence of dairy products without any pretreatment of the samples. Previously heat treatment and structural changes during coagulation have successfully been investigated in milk using fluorescence spectroscopy (Dufour and Riaublanc, 1997; Birlouez-Aragon et al., 1998; Herbert et al., 1999). Changes in fat and protein composition and structure have been characterized by the means of measuring the tryptophan and vitamin A fluorescence of cheeses during ripening (Dufour et al., 2000; Mazerolles et al., 2001) and for identification of different cheeses at a molecular level (Dufour et al., 2000; Herbert et al., 2000). Wold et al. (2002) demonstrated the potential of fluorescence spectroscopy for measuring the lightinduced oxidation, ascribed to the photodegradation of riboflavin.

Common to all these studies is that basic chemometric tools like Principal Component Analysis and Partial Least Squares Regression are applied for the evaluation of single excitation or emission fluorescence spectra. The multivariate approach increases the extracted information and is very useful when handling the fluorescence signal of complex food products. Even more information can be obtained, if the fluorescence measurements are not limited to single emission or excitation spectra. The possibilities when measuring whole fluorescence landscapes (excitation emission matrices) will be investigated here. New chemometric methods (Andersson and Bro, 2000) make it possible to handle fluorescence landscapes keeping the 2-dimensional data structure of each measurement. The techniques are known as $\mathrm{N}$-way or multiway chemometrics, and in the case of fluorescence signals, a 3-way (samples $\times$ excitation $\times$ emission) data analysis is an obvious choice. The advantage of the multiway analysis is that one can utilize the original and true structure in data, which can stabilize the decomposition of the data, and potentially increase the interpretability (Bro, 1996; Bro, 1997).

In the present study Parallel Factor analysis (PARAFAC) (Bro, 1997) is applied on the fluorescence landscapes of processed cheese exposed to light and varying temperature during storage. PARAFAC analysis of fluorescence data is previously used with success on model system of mixtures of fluorophores and in other food applications like sugar and fish (Bro, 1999; Baunsgaard et al., 2000a; Baunsgaard et al., 2000b; Pedersen et al., 2002) to investigate the present fluorescent compounds in complex matrices. PARAFAC is based on the decomposition of the fluorescence data represented in a three-way array, into a few spectral loadings expressing the common structure of the data. The feature of PARAFAC is that the retrieved loading spectra can be directly related to the original fluorescence characteristics of the present fluorophores, which means that the emission and excitation maximum of the loadings can be used in the interpretation and identification of the fluorophores (Bro, 1997).

Thus, the overall objective of the present investigation is to use multivariate analysis on fluorescence spectra keeping the 3-dimensional structure and extract information about the product at hand regarding age and storage conditions. This is pursued by using a nondestructive and rapid high-sensitive fluorescence method, which is simple to perform, and does not involve sample preparation.

\section{MATERIALS AND METHODS}

\section{Processed Cheese: Product and Storage Conditions}

The product and storage conditions are identical to the experimental plan used by Kristensen et al., 2001. A batch of processed cheese spread samples (density approximately $1.1 \mathrm{~g} / \mathrm{mL}$ ) with $65 \%$ fat in dry matter was obtained from Arla Foods amba, Denmark. The processed cheese was produced according to standard production of processed cheese and was constituted of bovine milk, starter culture, salt and emulsifier. After production the product was filled without any headspace $(140 \mathrm{~g})$ in transparent glass containers and sealed with a metal lid. The samples were stored for 10 months at three temperatures 5,20 , and $37^{\circ} \mathrm{C}$ and were exposed by placing the samples at a distance of approx. $55 \mathrm{~cm}$ from a fluorescent lamp or protected from light by wrapping the glass container in tin foil. The light source was fluorescent tubes (Phillips TLD 18/83 W) with a light intensity of $2000 \mathrm{~lx}$ as measured by a Topcon IM-1 illumination meter (Tokyo Kogaku Kikai K.K.). Samples were taken out at the beginning of the experiment and then after 14, 28, 56, 84, 112 and $256 \mathrm{~d}$. Only the $1 \mathrm{~cm}$ outer layer which had been in contact with the wall of the containers were used and each of the samples were taken from the glass jars by breaking the original seal prior to freezing at $-80^{\circ} \mathrm{C}$. The samples were frozen for a year before being thawed. Two cheese samples from each treatment were withdrawn for each analysis time.

\section{Fluorescence Spectroscopy and Sampling}

All samples were measured on a Perkin-Elmer LS 50B spectrometer equipped with a Front Surface Accessory and controlled with FLDM software. The stored cheese samples were mixed thoroughly before spread- 


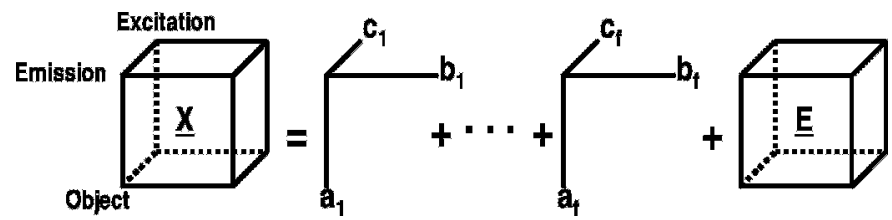

Figure 1. Illustration of the decomposition scheme into $f$ number of components of the PARAFAC model for the data array $\underline{\mathbf{X}}$. The cube $\underline{\mathbf{E}}$ represents the residual.

ing directly onto the quartz window of a powder cell, which was then assembled and placed in the light path in an angle of around $60^{\circ}$. The spectral range of the experiment was selected upon an exploratory basis. A preliminary investigation measuring excitation wavelengths from 200 to $600 \mathrm{~nm}$, and emission wavelengths from 220 to $800 \mathrm{~nm}$ on different cheeses were performed, and resulted in focusing on excitation wavelengths in the UV region. Strong fluorescence signals were obtained from the cheese samples in this area, leaving no signal from higher excitation and emission wavelengths when using this technique and set-up. The selected spectral range of the excitation wavelength was 240 to $360 \mathrm{~nm}$ with $20 \mathrm{~nm}$ intervals. Emission was obtained for every $\mathrm{nm}$ from 275 to $475 \mathrm{~nm}$. The slit width was $6 \mathrm{~nm}$ for excitation and $5 \mathrm{~nm}$ for the emission and a $1 \%$ attenuation filter was used.

It should be noted that the selected spectral range does not cover riboflavin fluorescence, which exhibit emission around $520 \mathrm{~nm}$ (Duggan et al., 1957), despite it would be an obvious compound to monitor throughout storage. However, the preliminary studies on cheese samples showed that no detectable signal was obtained in this spectral area when using the described measuring set-up.

\section{Data Analysis-PARAFAC}

PARAFAC decomposes the fluorescence spectra, into tri-linear components according to the number of fluorophores present the cheese samples (objects). The number of fluorophores present in the samples is equal to the minimal number of factors $(f=1, \ldots, F)$ needed to describe the fluorescence matrix $\underline{\mathbf{X}}$.

A graphical illustration of the decomposition of the data array $\underline{\mathbf{X}}$ is given in Figure 1. The object mode is expressed by the A-scores $\left(a_{1}, \ldots, a_{f}\right)$ and the two spectral loadings excitation and emission are expressed as B loadings $\left(b_{1}, \ldots, b_{f}\right)$ and $C$ loadings $\left(c_{1}, \ldots, c_{f}\right)$, respectively. The loadings in a spectral bilinear decomposition reflect the pure spectra of the fluorophores and the true underlying spectra can be recovered in the single components.
The principle behind the PARAFAC decomposition is to minimize the sum of squares of the residual $e_{i j k}$, see Equation 1.

$$
\begin{gathered}
x_{i j k}=\sum_{f=1}^{F} a_{i f} b_{j j} c_{k f}+e_{i j k} \\
(i=1, \ldots, I ; j=1, \ldots, J ; k=1, \ldots, K ; f=1, \ldots, F)
\end{gathered}
$$

The element $x_{i j k}$ represents the raw fluorescence excitation/emission spectra $(\underline{\mathbf{X}})$ of the stored cheese, where $i$ is the number of measured samples, $j$ is the number of excitation wavelengths, $k$ is the number of emission wavelengths and $f$ is the number of factors. $a_{. f}$ is the object score (magnitude of the fluorophore) for factor $f$ (first mode), $b_{\text {. } f}$ is the excitation loading for factor $f$ (second mode), and loading $c_{. f}$ express the emission spectra (third mode). $e_{i j k}$ is the residual $(\underline{\mathbf{E}})$ and contains the variation not captured by the PARAFAC model (Bro, 1997). Split half analysis is suggested for validation of PARAFAC models by Bro (1997). The idea of this strategy is to divide the data set into two halves and make a PARAFAC model on both halves. Due to the uniqueness of the PARAFAC model one will obtain the same result-same loadings in the nonsplitted mode e.g., excitation and emission mode-on both datasets, if the correct number of components is chosen.

\section{Calculating the PARAFAC Model}

The following sampling was performed: 45 samples $\times 2$ replicates $\times 2$ repetitions $=190$ samples. Seven samples were removed, as they were considered to be spectral outliers based on a preliminary data inspection and resulted in a total of 183 samples. The preliminary PARAFAC modelling indicated that nonnegativity constraints on all three modes (samples, excitation, and emission) were necessary. Validation of the PARAFAC modelling was performed with split half test, based on replicated samples, i.e. not splitting of the repetitions.

In addition to the split-half experiment, the residuals were inspected, and the results were judged, interpreted and compared with external knowledge.

All calculations were performed in Matlab version 6.1 (MathWorks, Inc.) with the $\mathrm{N}$-way Toolbox (Andersson and Bro, 2000) and the PLS Toolbox (www.Eigenvector.com).

\section{RESULTS AND DISCUSSIONS}

The fluorescence landscapes of two cheese samples are shown in Figure 2. The two samples represent the extremes in the experimental plan, i.e., a fresh cheese sample (a) and a cheese sample stored under the most severe conditions (b). The highest fluorescence peak for 


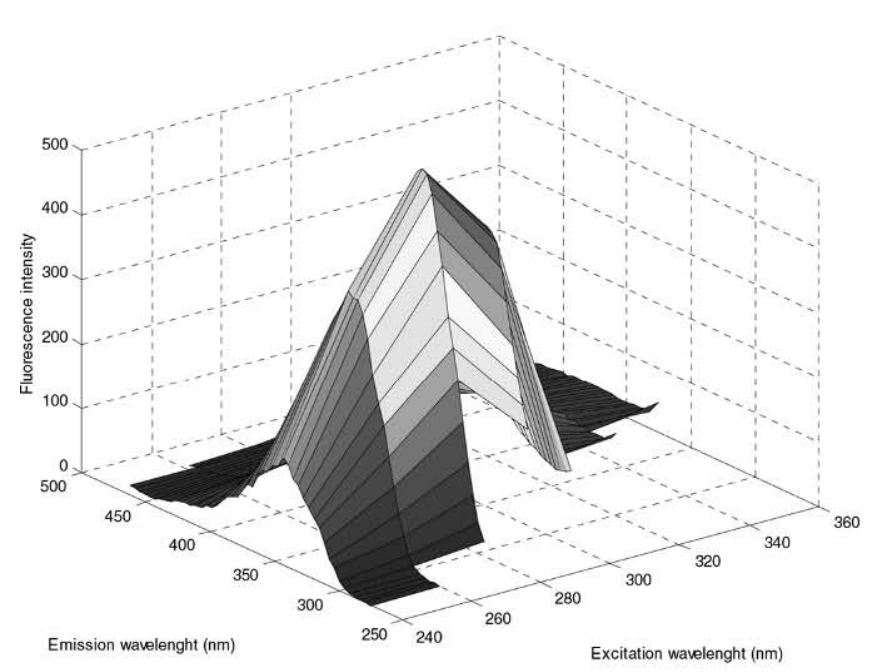

A

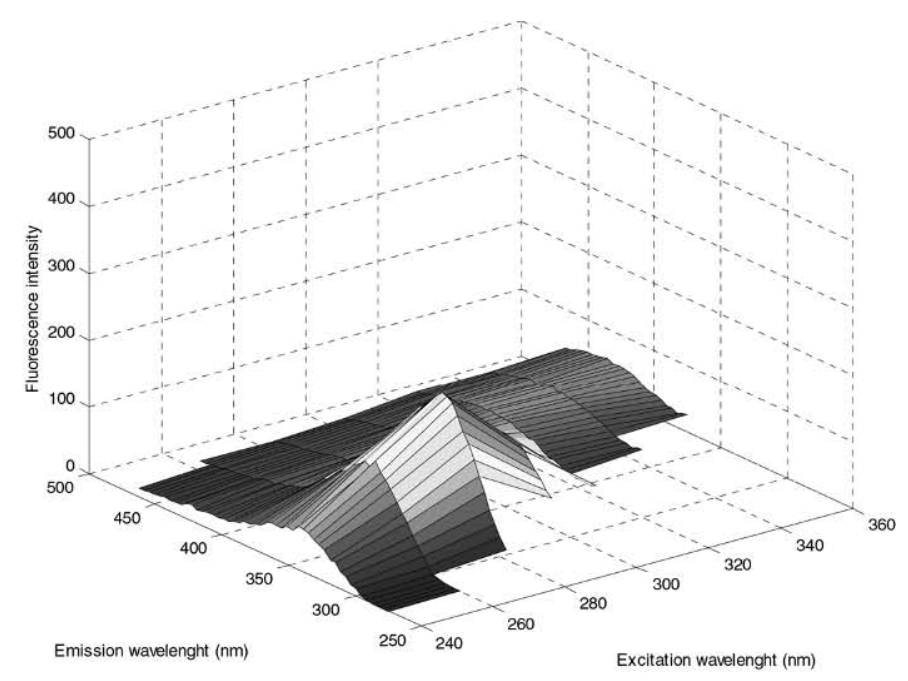

B

Figure 2. Three dimensional plot of fluorescence landscapes of processed cheese samples. a) fresh cheese sample, and b) cheese sample stored in $259 \mathrm{~d}$ at $37^{\circ} \mathrm{C}$ exposed to light.

both samples is seen with excitation around $280 \mathrm{~nm}$ and emission around $350 \mathrm{~nm}$, with a significant higher and apparently broader signal from the fresh cheese. The excitation and emission characteristics indicate that the fluorescence peak corresponds to tryptophan fluorescence, which is reported to have excitation/emission wavelength maximum at $285 / 365 \mathrm{~nm}$ in pure solutions (Duggan et al., 1957), and previously measured in cheese products with excitation $290 \mathrm{~nm}$ and emission from 305-400 nm (Herbert et al., 2000; Dufour et al., 2001; Mazerolles et al., 2001). Apart from this major peak, a vague peak is observed in the higher wavelength region with excitation around 320 to $360 \mathrm{~nm}$ and emission round 400 to $460 \mathrm{~nm}$, especially for the cheese sample stored for $259 \mathrm{~d}$.

The aforementioned patterns in the fluorescence landscapes were investigated further by the use of PARAFAC analysis with the objective to resolve the fluorescence signal into the contributions of each of the fluorescent compounds present in the set of samples, i.e. estimate the excitation and emission profiles of fluorophores directly from the three-dimensional fluorescence landscapes. PARAFAC models of the fluorescence data were estimated with one to five components, but the four-component model was chosen based on split half analysis (Bro, 1997). A high explained variation of 99.76\% is captured by the PARAFAC model, and the resulting PARAFAC components are shown in Figure 3 . The model indicates that four different fluorophores are present in the cheese samples with the excitation and emission profiles shown in the figure. The excita- tion/emission maximum for the two compounds are 300/ $347 \mathrm{~nm}$ and 280/339 $\mathrm{nm}$, respectively, as listed in Table 1 . The loading profiles of the second PARAFAC component corresponds quite well with the characteristics of tryptophane, whereas the excitation maximum of the first component seems a little too high for tryptophan. Having the rather low resolution of $20 \mathrm{~nm}$ in the excitation mode in mind, and knowing that the fluorescence properties of protein-bound amino-acids are known to be affected by the structure of protein (Lakowicz, 1999), we dare to suggest that the first PARAFAC components is also due to tryptophan fluorescence, but simply shifted due to inclusion to different protein structures.

The score values in the first column of Figure 3 represent the concentration mode for each of the fluorophores, and since the excitation and emission loadings are normalized when calculating the PARAFAC model, the contribution for each of the components can be compared to the overall variation based on the level of the scores. The score values are arranged so the development of the fluorophores easily can be caught throughout the storage time. Looking at the two proposed tryptophan components, a significant decrease is observed throughout the storage period for the samples stored at $37^{\circ} \mathrm{C}$. This shows that alterations in the protein structure, monitored by the decrease in tryptophan fluorescence, somehow can reflect the conditions of the cheese samples during storage. The samples exposed to light during storage show a systematically higher tendency to be degraded throughout the storage than the samples stored in the dark. Compared with the 

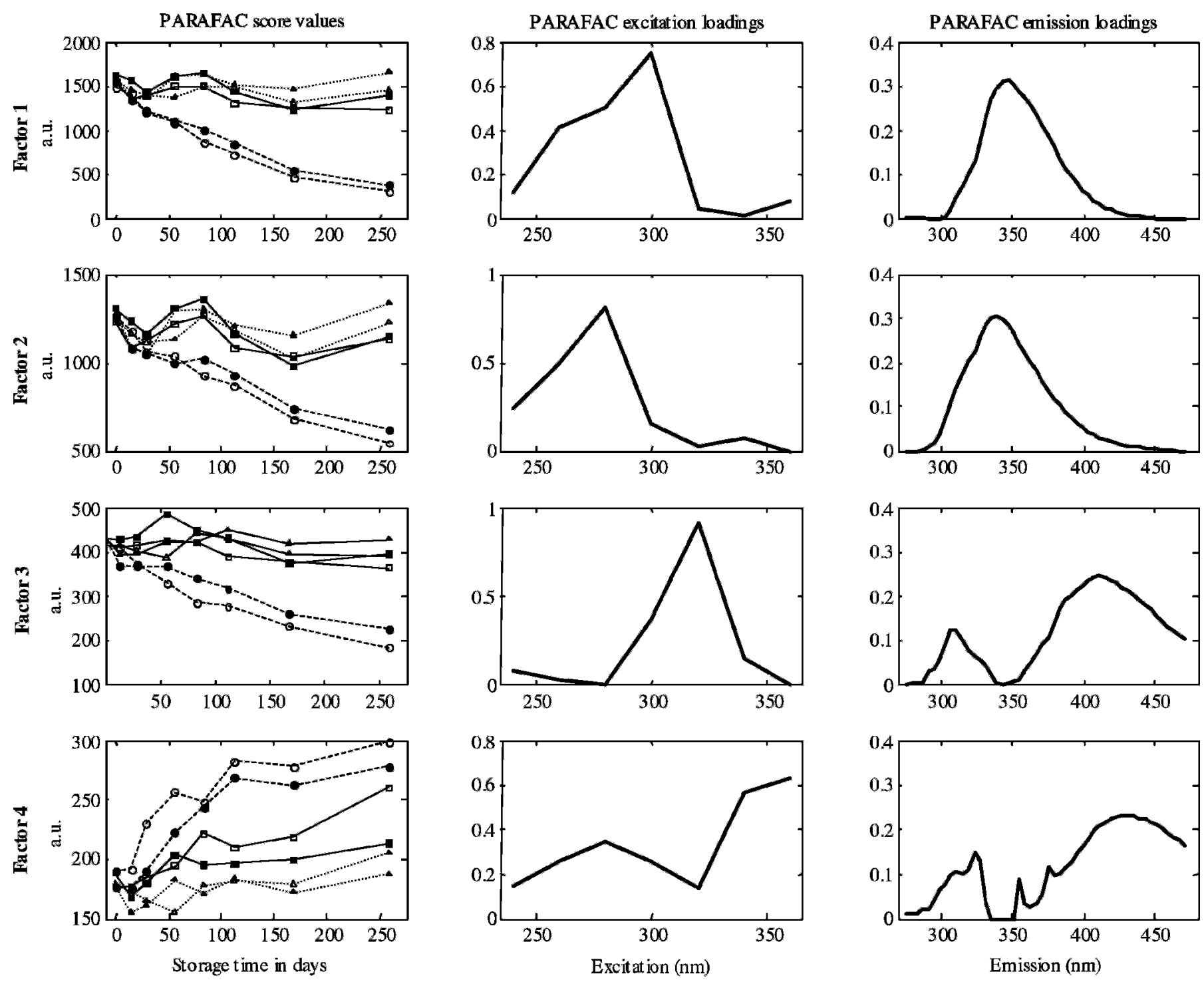

Figure 3. A- (scores), B- (excitation), and C- (emission) loadings of a four component PARAFAC model, based on the fluorescence landscapes of 183 processed cheese samples. Samples stored at $5^{\circ} \mathrm{C}$ are indicated with triangles, and connected with dotted line (…). Samples stored at 20 and $37^{\circ} \mathrm{C}$ are shown with squares and dashed line (- - ) and circles connected with a full line (—), respectively. Open signs represent samples stored in light, and filled sign illustrates storage in darkness.

effect of different temperatures, the light exposure seems negligible for the two tryptophan components, though. The same storage experiment showed a similar tendency of light exposure having little, if any influence on the browning of cheese (Kristensen et al., 2001), and thereby indicate that the observed differences in the protein structure are somehow related to the browning reaction i.e. the formation of Maillard products from the protein and lipid oxidation products in cheese, even though tryptophan itself may not be part of the browning reaction scheme. As indicated by the first visual inspection of the fluorescence landscapes, the level of the score values for the two first components are much higher than the third and fourth component, simply showing that the development in the tryptophan signal represents the major variation in the fluorescence data.

The development of the third estimated fluorophore (score values of the third PARAFAC component) shows a similar pattern as the decrease in the tryptophan signal. Thus, the cheese samples stored at $37^{\circ} \mathrm{C}$ contain less of this component throughout the storage, especially the samples exposed to light during storage. Comparing the fluorescence profiles seen in Figure 3 and the excitation/emission wavelength maximum of 320/ $411 \mathrm{~nm}$ (Table 1) with observed maximum of 325/470 $\mathrm{nm}$ in pure solution reported (Duggan et al., 1957) and 
Table 1. Excitation and emission maximum of four components in the PARAFAC model of 45 different cheese samples.

\begin{tabular}{lll}
\hline Component & $\lambda_{\max }(\mathrm{nm})$ & \\
Excitation & Emission \\
\hline 1 & 300 & 347 \\
2 & 280 & 339 \\
3 & 320 & 411 \\
4 & 360 & 431 \\
\hline
\end{tabular}

$322 / 412 \mathrm{~nm}$ for dairy products (Dufour et al., 2001; Herbert et al., 2000), vitamin A is an obvious suggestion for the third component. This is underlined by the fact, that the observed decrease in vitamin A fluorescence signal throughout the storage period corresponds well to reported vitamin A degradation during light exposure in dairy products (Whited et al., 2000).

The fourth PARAFAC component reveals an opposite and very interesting trend in the score values, as seen in Figure 3. The level of this fluorophore increases throughout the storage period, especially for the cheese samples stored in light. The excitation and emission loadings look somewhat noisier with several small peaks, probably caused by the fact that the fluorescence signal is very low, as can be seen from the levels of the score values. Scattering effects might be the reason for the extra emission peak observed around $320 \mathrm{~nm}$ for both the third and the fourth component. The identification of the fourth fluorescent compound, showing an increase signal during the oxidation of the cheese samples, give rise to more doubt. Taking the increasing concentration of the fourth component throughout storage in consideration, it is obvious to suggest that the fourth component can be attributed to some kind of oxidation product. So-called "Advanced Maillard Products" in milk samples have been reported (BirlouezAragon et al., 1998) to excite around $350 \mathrm{~nm}$ with emission at $440 \mathrm{~nm}$, which is almost identical to the peak observed in the fourth component. Another suggestion could be that the fourth component is a secondary oxidation product developed when carbonyl compounds produced by lipid oxidation interacts, as reported by Dillard and Tappel (1971) with a fluorescent compound from lipid peroxidation with excitation maximum at $360 \mathrm{~nm}$, and emission maximum at $430 \mathrm{~nm}$, which is even closer to the fluorescent characteristics of the fourth component. Finally, Stapelfeldt and Skibsted (1994), demonstrated that the reaction between secondary lipid oxidation products from milk products and $\beta$ lactoglobulin in a model system yielded a fluorescent condensation products with excitation/emission maximum at 350/410 nm, which could also form an educated guess for identification of the fourth PARAFAC component.

\section{CONCLUSIONS}

This exploratory study of processed cheese demonstrates the potential of fluorescence spectroscopy and chemometrics applied to the analysis of dairy products. The rapid fluorometric analysis reveals information at a molecular level about the stability of the cheese when exposed to manufacture handling stress like light and temperature changes. PARAFAC analysis provides a unique mathematical decomposition of four fluorescent compounds present in the cheese samples all showing a change in the fluorescence signal corresponding the storage time and the grade of oxidation.

The fluorescent signal from the processed cheese samples is suggested to derive from tryptophan, vitamin A and an oxidation product. Thus, the suggested analytical method provides a fast and simultaneous determination of the fluorescence level of all these compounds. The observed results still remain to be validated with chemical reference analyses in order to proof the identification of the fluorophores, but this investigation certainly underlines the potential of fluorescence spectroscopy in combination with chemometrics, as a fast, nondestructive innovative method, that can be applied to dairy products for monitoring oxidation, screening studies and perhaps in development of new fast quantitative analyses of vitamin $\mathrm{A}$.

\section{ACKNOWLEDGMENTS}

This study is partly sponsored by an EU innovation programme, OPUS (IN30905 I), with the aim to adapt and apply advanced fluorescence measurement techniques for online analysis to traditional food industries. Povlsen wish to thank FØTEK 3 (93S-2444-Å01-00100) for financial support. Furthermore the authors wish to thank Joanna Zeppelin and Vibeke Birk from Arla Foods for making this publication possible, and for carrying out all the experimental work. Finally we are grateful to Professor Rasmus Bro for valuable discussion.

\section{REFERENCES}

Andersson, C. A., and R. Bro. 2000. The N-way Toolbox for MATLAB. Chemometrics Intell. Lab. Syst. 52:1-4.

Baunsgaard, D., C. A. Andersson, A. Arndal, and L. Munck. 2000a. Multi-way chemometrics for mathematical separation of fluorescent colorants and colour precursors from spectrofluorimetry of beet sugar and beet sugar thick juice as validated by HPLC analysis. Food Chem. 70:113-121.

Baunsgaard, D., L. Munck, and L. Norgaard. 2000b. Analysis of the effect of crystal size and color distribution on fluorescence measurements of solid sugar using chemometrics. Appl. Spec. 54:1684-1689.

Birlouez-Aragon, I., M. Nicolas, A. Metais, N. Marchond, J. Grenier, and D. Calvo. 1998. A rapid Fluorimetric Method to estimate the heat treatment of liquid Milk. Int. Dairy J. 8:771-777. 
Bro, R. 1996. Multi-way Calibration Multi-Linear PLS. J. Chemometrics. 10:47-62.

Bro, R. 1997. PARAFAC. Tutorial and applications. Chemometrics Intell. Lab. Syst. 38:149-171.

Bro, R. 1999. Explorative study of sugar production using fluorescence spectroscopy and PARAFAC analysis. Chemometric Intell. Lab. Syst. 46:133-147.

Dillard, C. J., and A. L. Tappel. 1971. Fluorescent Products of Lipid Peroxidation of Mitochondria and Microsomes. Lipids. 6:715-721.

Dufour, E., M. F. Devaux, and S. Herbert. 2001. Delineation of the structure of soft cheeses at the molecular level by fluorescence spectroscopy-relationship with texture. Int. Dairy J. 11:465473.

Dufour, E., G. Mazerolles, M. F. Devaux, G. Duboz, M. H. Duployer, and N. M. Riou. 2000. Phase Transition of triglycerides during semi-hard cheese ripening. Int. Dairy J. 10:81-93.

Dufour, E., and A. Riaublanc. 1997. Potentiality of spectroscopic methods for the characterisation of dairy products. I. Front-face fluorescence study of raw, heated and homogenised milks. Lait $77: 657-670$

Duggan, D. E., R. L. Bowman, B. B. Brodie, and S. Udenfriend. 1957. A Spectrophotofluorometric Study of Compounds of Biological Interest. Arch. Biochem. and Biophys. 16:1-14.

Emmons, D. B., G. J. Paquettte, D. A. Froehlich, D. C. Beckett, H. W. Modler, G. Butler, Brackenbridge, and G. Daniels. 1986. Oxidation of butter by low intensities of fluorescent light in relation to retail stores. J. Dairy Sci. 69:2437-2450.

Herbert, S., A. Riaublanc, B. Bouchet, D. J. Galllant, and E. Dufour. 1999. Fluorescence Spectroscopy Investigation of Acid- or RennetInduced Coagulation of Milk. J. Dairy Sci. 82:2056-2062.

Herbert, S., N. M. Riou, M. F. Devaux, A. Riaublanc, B. Bouchet, D. J. Galllant, and E. Dufour. 2000. Monitoring the identity and the structure of soft cheeses by fluorescence spectroscopy. Lait. 80:621-634

Kristensen, D., E. Hansen, A. Arndal, R. A. Trinderup, and L. H. Skibsted. 2001. Influence of light and temperature on the colour and oxidative stability of processed cheese. Int. Dairy J. 11:837-843.

Kristensen, D., and L. H. Skibsted. 1999. Comparison of Three Methods Based on Electron Spin Resonance Spectrometry for Evaluation of Oxidative Stability of Processed Cheese. J. Agric. Food Chem. 47:3099-3104.

Lakowicz, J. R. Protein fluorescence. Pages 446-485 in Principles of fluorescence spectroscopy. Kluwer Academic/plenum Publishers, New York.

Mazerolles, G., M. F. Devaux, G. Duboz, M. H. Duployer, N. M. Riou, and E. Dufour. 2001. Infrared and fluorescence spectroscopy for monitoring protein structure and interaction changes during cheese ripening. Lait. 81:509-527.

Pedersen, D. K., L. Munck, and S. B. Engelsen. 2002. Screening for dioxin contamination in fish oil by PARAFAC and N-PLSR analysis of fluorescence landscapes. J. Chemometrics. In press.

Stapelfeldt, H., and L. H. Skibsted. 1994. Modification of $\beta$-lactoglobulin by aliphatic aldehydes in aqueous solution. J. Dairy Res. 61:209-219.

Sunesen, L. O., P. Lund, J. Sørensen, and G. Hølmer. 2002. Development of volatile compounds in processed cheese during storage. Lebensmittel-Wissenschaft Und-Technologie. 35:128-134.

Whited, L. J., B. H. Hammond, K. W. Chapman, and K. J. Boer. 2002. Vitamin A Degradation and Light-Oxidized Flavor Defects in Milk. J. Dairy Sci. 85:351-354.

Wold, J. P., K. Jørgensen, and F. Lundby. 2002. Nondestructive Measurement of Light-induced Oxidation in Dairy Products by Fluorescence Spectroscopy and Imaging. J. Dairy Sci. 85:1693-1704. 